\title{
Observation of geodesic acoustic modes (GAMs) and their radial propagation at the edge of TEXTOR tokamak
}

\author{
Y. $\mathrm{Xu}^{1}$, I. Shesterikov ${ }^{1}$, M. Van Schoor $^{1}$, M. Vergote ${ }^{1}$, \\ R. R. Weynants ${ }^{1}$, A. Krämer-Flecken ${ }^{2}$, S. Zoletnik ${ }^{3}$, S. Soldatov ${ }^{2}$, \\ D. Reiser ${ }^{2}$, K. Hallatschek ${ }^{4}$, C. Hidalgo ${ }^{5}$, and the TEXTOR Team \\ ${ }^{1}$ Laboratoire de Physique des Plasmas - Laboratorium voor Plasmafysica, \\ Association 'Euratom-Belgian state', \\ Ecole Royale Militaire - Koninklijke Militaire School, \\ Trilateral Euregio Cluster, B-1000 Brussels, Belgium \\ 2 Institute of Energy and Climate Research - Plasma Physics, \\ Forschungszentrum Jülich GmbH, Association EURATOM-FZJ, \\ Trilateral Euregio Cluster, D-52425 Jülich, Germany \\ 3 KFKI RMKI, Association Euratom, \\ PO Box 49 H-1525 Budapest, Hungary \\ 4 Max-Planck-Institut für Plasmaphysik, D-85748 Garching, Germany and \\ ${ }^{5}$ Laboratorio Nacional de Fusion, Association EURATOM-CIEMAT, 28040 Madrid, Spain
} (Dated: July 26, 2011) 


\begin{abstract}
The electrostatic potential and density fluctuations have been measured at the edge of TEXTOR tokamak by two toroidally distant Langmuir probe arrays. The geodesic acoustic mode (GAM) zonal flows are observed in potential fluctuations with a toroidal and poloidal symmetric structure. The GAM frequency, $f_{G A M}$, changes monotonically with the local temperature and is close to the frequency-dispersion predicted by theories. Bispectral analysis shows clear nonlinear coupling between the GAM and broadband ambient turbulence. The GAM packet has a narrow radial extent with $k_{r} \simeq(0.5-0.7) \mathrm{cm}^{-1}$ and exhibits explicitly a radially outward propagation. Furthermore, the radial correlation structure of GAMs and their radial propagation have been investigated in a wide range of parameters by varying plasma density $\left(\bar{n}_{e 0}=(1.5-3.0) \times 10^{19} \mathrm{~m}^{-3}\right)$ and edge safety factor $(5.0 \leq q(a) \leq 5.9)$. It is found that the magnitude of the GAM correlations reduces remarkably with the increase of the plasma density as approaching to the density-limit, while the radial wavelength of GAMs only decreases slightly in higher density and larger $q(a)$ discharges. With increasing plasma density, the radial propagating phase speed of GAMs is strongly reduced along with the drop of the local temperature. The results provide new evidence on the propagation properties of GAM zonal flows.
\end{abstract}




\section{INTRODUCTION}

Characterizing the feature of zonal flows (ZFs) is still an important subject in fusion plasmas as it has been predicted by theories that the ZFs may play a key role in regulating turbulence level and associated transport via nonlinearly transferring energy from ambient turbulence and in turn, suppressing turbulence by shear effects on fluctuations [1]. As a branch of ZFs, the geodesic acoustic mode (GAM) with finite frequencies has been observed in a variety of toroidal devices [2-11]. According to theories $[1,12,13]$, the GAM is generated due to the linear coupling (by toroidal curvature effects) between the poloidal/toroidal mode number $m / n=0 / 0$ electrostatic potential (or electric field) and the $m / n=1 / 0$ sideband density (or pressure) perturbations. However, hitherto the radial propagation of GAMs has not yet been clearly described. In earlier work [12, 13], the GAMs turned out to be standing

waves with a frequency-dispersion $f_{G A M} \simeq C_{s} \sqrt{2+1 / q^{2}} / 2 \pi R$, where $C_{s}, R$ and $q$ are the sound speed, major radius and safety factor, respectively. In recent work, the radial propagation of GAMs and GAM eigenmodes have been discussed by several authors [14-18], but no general rules have been given for the direction and velocity of the GAM propagation. The experimental observation on the GAM propagation has rarely been reported either. Only in the HL-2A tokamak the statistical analysis of the radial wavenumber spectrum gives hints that the GAM packet propagates in radially outward direction with approximately the same group and phase velocity [11]. In the JFT-2M tokamak and TJ-II stellarator a sign of radially propagating GAMs or zonal flows (below $20 \mathrm{kHz}$ ) has also been commented [7, 19].

In this paper we present additional evidence of GAMs detected by two toroidally spaced Langmuir probe arrays at the edge of TEXTOR tokamak. Their spatial structure, frequencydispersion and nonlinear coupling with ambient turbulence are all characterized. Moreover, we report direct observations that the GAMs propagate radially outwards at different phase velocities in different plasma discharge conditions.

The paper is organized as follows. In section 2 the experimental set-up is described. Section 3 presents the experimental results and discussion, and section 4 gives the conclusion. 


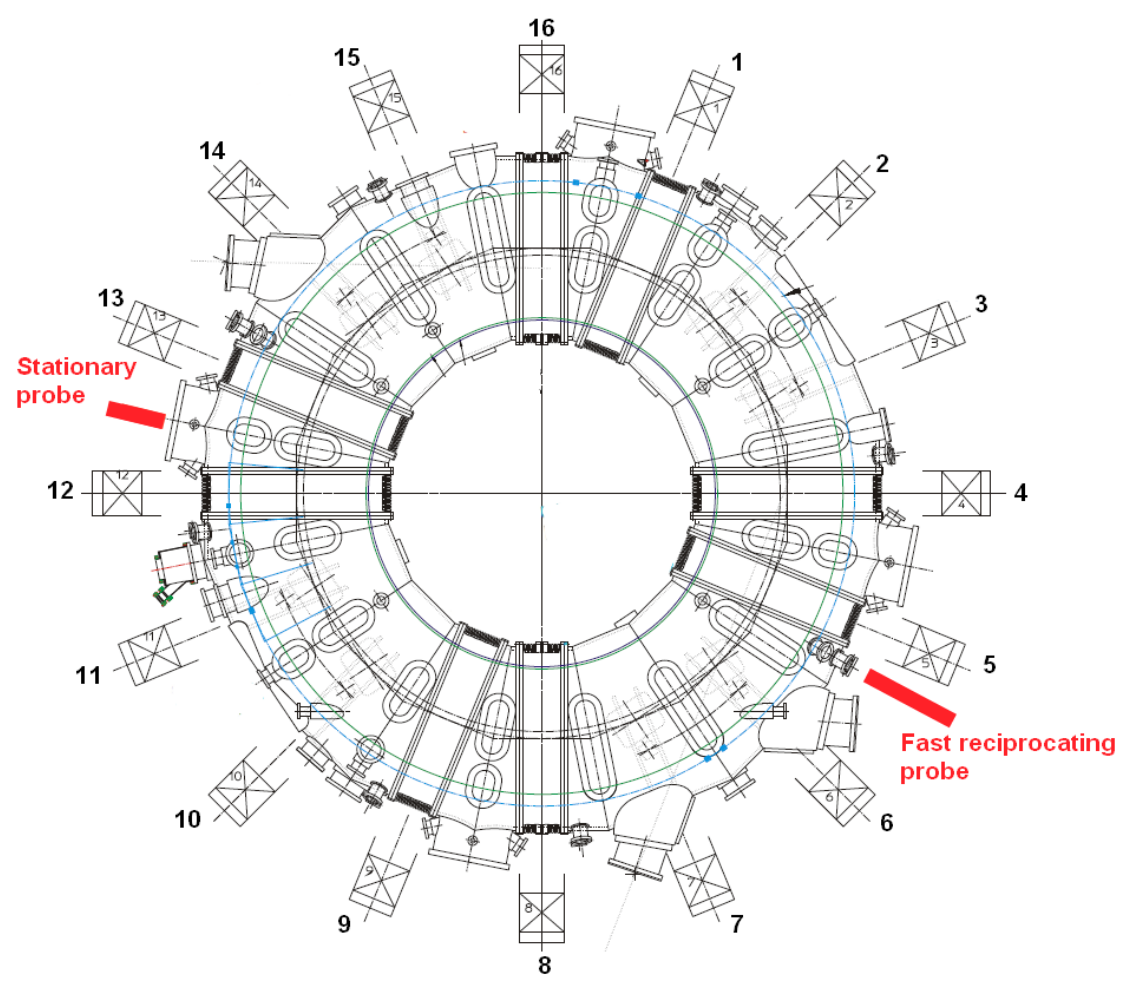

FIG. 1: Top view of TEXTOR tokamak showing two probe systems toroidally spaced (over a distance $\approx 7 \mathrm{~m}$ ) to detect the long-range correlation of edge fluctuations. One probe array is fast reciprocating (section 5/6) and the other is stationary (section 12/13).

\section{EXPERIMENTAL SET-UP}

The experiments were carried out in ohmic deuterium discharges in the TEXTOR tokamak with the major radius $R=175 \mathrm{~cm}$ and minor radius $a \approx 48 \mathrm{~cm}$. Typical plasma current was $I_{p}=200-250 \mathrm{kA}$, toroidal magnetic field $B_{T}=1.9-2.25 \mathrm{~T}$, central line-averaged density $\bar{n}_{e}=(1.5-3.0) \times 10^{19} \mathrm{~m}^{-3}$ and the edge safety factor $q(a)=5.0-6.3$. In order to identify one of key features of the GAM zonal flow, i. e., the axisymmetric $n=0$ mode structure, we measured the long-distance toroidal correlation of edge fluctuations by two Langmuir probe arrays. Figure 1 shows the top view of TEXTOR, where two movable probe arrays were installed at two approximately opposite locations of the torus (over a toroidal distance $\sim 7.0 \mathrm{~m}$ ). Both arrays were mounted in the midplane of the low-field side and equipped to measure the floating potential $\left(V_{f}\right)$ and ion saturation current $\left(I_{s}\right)$ simultaneously. In each discharge, one probe array is stationary (in section 12/13) while the other is fast reciprocating (in section 5/6). From shot to shot, the radial position of the stationary probe can be 
altered. The fluctuation data were digitized at a rate of $500 \mathrm{kHz}$.

\section{RESULTS AND DISCUSSION}

\subsection{Properties of GAM zonal flows}

Typical results of long-distance correlations in edge turbulence and GAM zonal flows are illustrated in Fig. 2. Shown in Fig. 2(a) is the time trace of the fast probe, moving from the scrape-off layer (SOL) into the plasma edge. For the stationary probe, a schematic is shown for its radial position at $\mathrm{r} \approx 46 \mathrm{~cm}$ inside the limiter as a reference probe. The toroidal cross-correlation between the signals of $x(t)$ and $y(t)$, measured respectively on the stationary and the fast reciprocating probe, is defined as $C_{x y}(\tau)=\langle[x(t)-\bar{x}][y(t+\tau)-$ $\bar{y}]\rangle / \sqrt{\left\langle[x(t)-\bar{x}]^{2}\right\rangle\left\langle[y(t)-\bar{y}]^{2}\right\rangle}$, where $\tau$ is the time lag and $\langle\ldots\rangle$ denotes ensemble averaging. The corresponding time evolutions of the contour plot of $C_{x y}(\tau)$ of $I_{s}$ and $V_{f}$ fluctuations are depicted in Fig. 2(b) and (c), respectively. One can clearly see that in Fig. 2(b) there is nearly no correlation in $I_{s}$ fluctuations while in Fig. 2(c) a large cross-correlation in $V_{f}$ fluctuations occurs when the two toroidally separated probes are localized around the same radial position inside the last closed flux surface (LCFS). These results are in accordance with the theoretical prediction of GAM zonal flows $[1,12,13]$ : (i) the GAM is predicted to have an $m=1$ mode structure $(n=0)$ in density perturbation, thus the present probe arrays both installed in the outer midplane $\left(\theta=0^{\circ}\right.$ for both $)$ cannot detect the GAM signature in $I_{s}$ fluctuations ( $\propto$ density fluctuations). In TEXTOR, the $m=1$ component of GAMs in density fluctuations has been observed by the correlation reflectometer using two antennas which were located on the top $\left(\theta=90^{\circ}\right)$ and the equatorial plane $\left(\theta=0^{\circ}\right)$, respectively [10]; (ii) the long-range correlation seen in $V_{f}$ fluctuations $\left(\tilde{V}_{f}\right)$ is consistent with the prediction of an $m=n=0$ structure for GAMs in electrostatic potential perturbations. As shown in Fig. 2(c), when the two toroidally distant probes are around the same radial location $(r \approx 46 \mathrm{~cm})$, the maximum cross-correlation in $\tilde{V}_{f}$ appears with roughly a zero time lag $\tau \approx 0$ (see also in Fig. 2(e)), indicating a toroidal mode $n=0$. In this study, the poloidally uniform structure $(m=0)$ of GAMs has also been verified in the cross-correlation of $\tilde{V}_{f}$ by poloidally spaced probe pins over a distance of $12 \mathrm{~mm}$.

In Fig. 2(c) it is noticed that on both sides of the stationary probe, $r \geq 46 \mathrm{~cm}$ and 


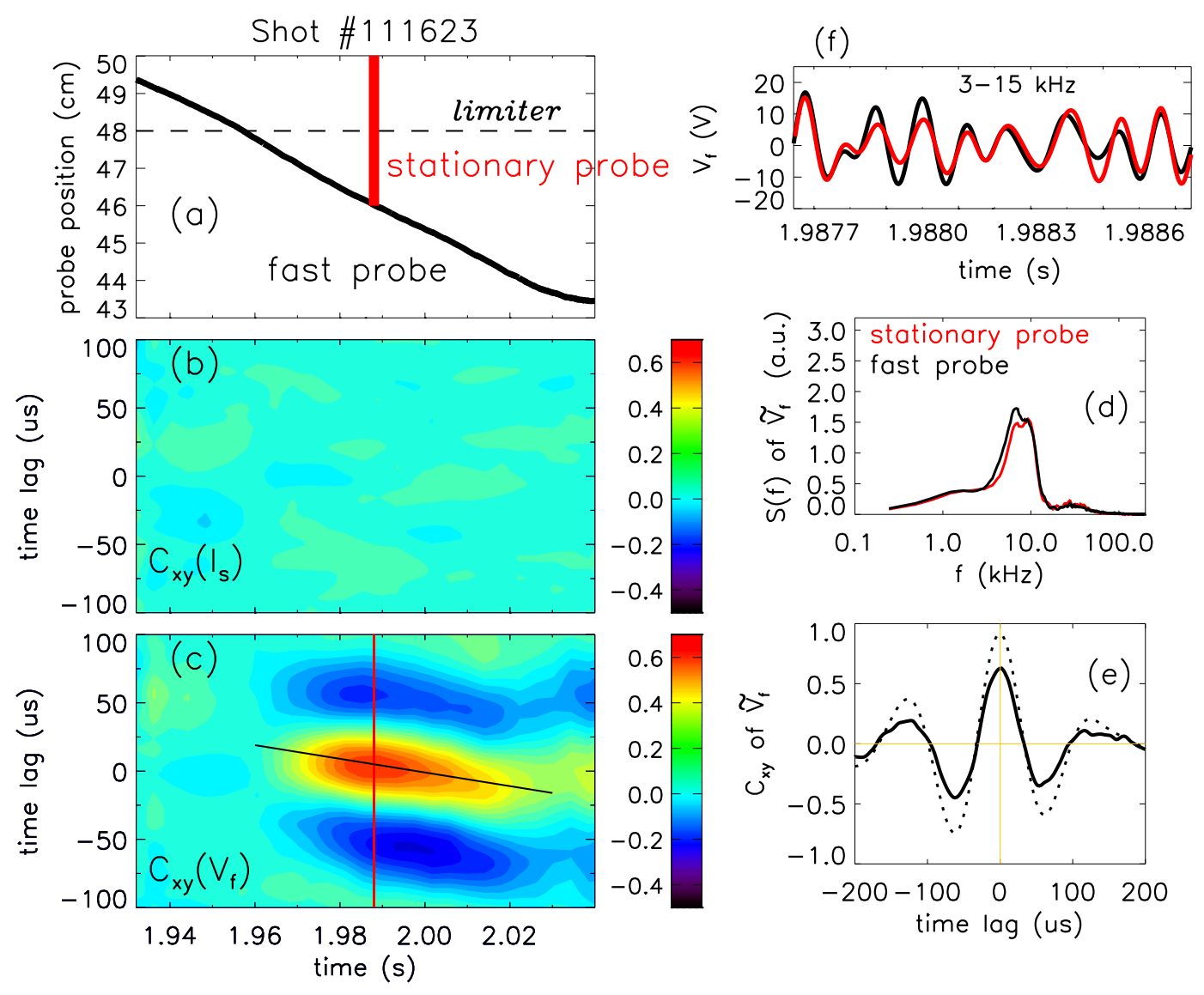

FIG. 2: (a) Time trace of fast/stationary probes; (b) contour plot of cross-correlation between $I_{s}$ fluctuations measured by two distant probe arrays; (c) contour plot of cross-correlation between $V_{f}$ fluctuations measured by two distant probe arrays. The vertical red line indicates the time when the two probes are at the same radial location; (d) power spectrum of $\tilde{V}_{f}$ detected by fast/stationary probe at the same radial location $(r \approx 46 \mathrm{~cm})$; (e) cross-correlation function on $\tilde{V}_{f}$ measured by the fast/stationary probe at $r \approx 46 \mathrm{~cm}$. The dotted curve is the cross-correlation computed from filtered $\tilde{V}_{f}$ signals in a frequency range of $3-15 \mathrm{kHz}$; (f) waveforms of $\tilde{V}_{f}$ around the same flux surface $(r \approx 46 \mathrm{~cm})$ in two toroidally distant positions (the red is for the stationary probe and the black is for the fast probe). Both signals are filtered in the frequency range of 3-15 kHz.

$r \leq 46 \mathrm{~cm}$, the $C_{x y}(\tau)$ displays clearly nonzero time lags, which vary depending on the radial position of the fast probe relative to the stationary (reference) one. On the left side $(r \geq 46 \mathrm{~cm})$ the maximum $C_{x y}$ turning up at $\tau>0$ reveals a radial motion of waves from the stationary probe toward outside, while on the right side $(r \leq 46 \mathrm{~cm})$ the maximum $C_{x y}$ at $\tau<0$ indicates a radial motion from the inside toward the stationary probe posi- 
tion. Thus, the contour plot of $C_{x y}(\tau)$ explicitly exhibits a radially outward propagation of the mode structure, as marked by a black straight line. Figure 2(d) plots the autopower spectrum of $\tilde{V}_{f}$ measured by the fast/stationary probe at the maximum $C_{x y}$ location $(r \approx 46 \mathrm{~cm})$. The spectra on both probes are very similar and a prominent peak is found around $f \approx 8.5 \sim 9.0 \mathrm{kHz}$, suggesting that the observed long-range correlation is dominated by coherent mode oscillations. To view in more detail, the $C_{x y}(\tau)$ measured at $r \approx 46 \mathrm{~cm}$ and the time evolution of the $V_{f}$ signals, filtered in a frequency range of $3-15 \mathrm{kHz}$ on the two toroidlally distant probes around the same radial position, are drawn in Figs. 2(e) and (f), respectively. In Fig. 2(e) one can see a periodic variation in the cross-correlation as a function of lag time. The period $(\approx 120 \mu \mathrm{s})$ is coincident with the peaked mode frequency shown in the power spectrum. The waveforms in Fig. 2(f) further verify that the zonal potentials on the two probes alter temporally in-phase at the same magnetic flux surface. In the experiment, the radial position of the stationary probe has been changed from $\rho=r / a=0.95$ to 0.97 in different shots. It appears that the maximum $C_{x y}$ on $\tilde{V}_{f}$ always takes place at the location where the two probes are around the same flux surface.

Note that in Fig. 2 the $C_{x y}(\tau)$ is calculated by the cross-covariance of $x(t)$ and $y(t)$ signals normalized by their standard deviations. Whereas the local turbulence (high-frequency ambient turbulence) is not correlated over the long toroidal distance in the covariance, their contribution is taken into account in the denominator. The value of $C_{x y}(\tau)$ is thus underestimated. To testify this, we computed the $C_{x y}(\tau)$ of $\tilde{V}_{f}$ on the two probes by filtering the non-GAM components in the $V_{f}$ signals, i. e., band-pass $V_{f}$ in a frequency range of 3-15 $\mathrm{kHz}$. The maximum $C_{x y}(\tau)$ at $r \approx 46 \mathrm{~cm}$ is plotted in Fig. 2(e) by a dotted curve. Obviously, by removing the impact of ambient turbulence, the value of $C_{x y}(\tau)$ is largely enhanced and approaches to unity. Nevertheless, except for the absolute value, we found that all other features of the cross-correlation are nearly the same, which will be further illustrated in Fig. 5 as well. Figure 2(f) also shows that the GAM amplitude on $\tilde{V}_{f}$ is not constant in time, indicating that the energy of GAM zonal flows is modulated instantaneously due to strong interaction between GAMs and background turbulence.

To examine the consistency of the experimentally observed mode frequency with the GAM oscillation, it is necessary to compare it with the theoretical expectation $[1,12,13,20]$, i. e., $f_{G A M} \simeq C_{s} \sqrt{2+1 / q^{2}} / 2 \pi R$ with $C_{s}=\sqrt{\gamma\left(T_{e}+T_{i}\right) / M_{i}}$ and the adiabatic coefficients $\gamma$ ranged from 1 to $5 / 3$ [20]. For the present deuterium discharge shown in Fig. 2, the lo- 


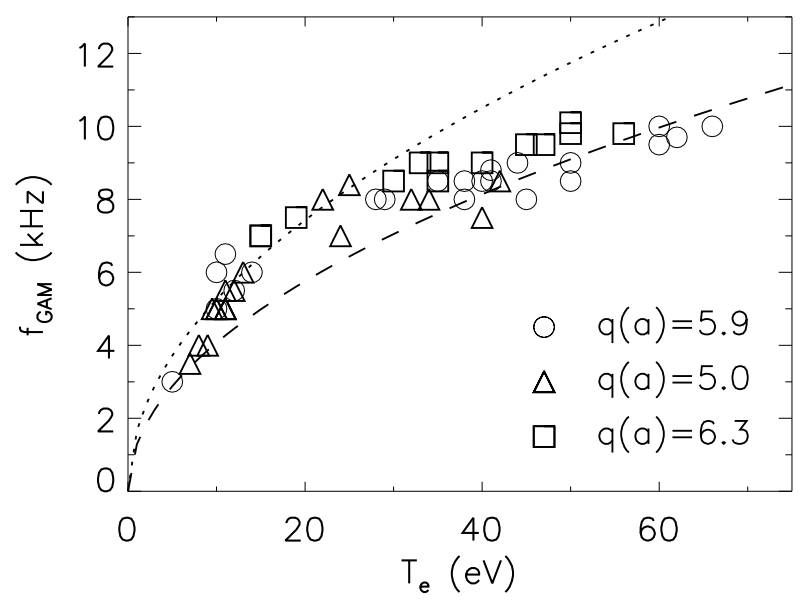

FIG. 3: The GAM frequencies as a function of temperature measured in three different safety factor plasmas at the edge of TEXTOR. The dashed $(\gamma=1)$ and dotted line $(\gamma=5 / 3)$ indicate the expected dispersion relations by the theory with two different adiabatic coefficients $(\gamma)$.

cal electron temperature at $\mathrm{r}=46 \mathrm{~cm}$ measured by the fast probe array (using triple probe model [21]) is $T_{e} \simeq 25 \mathrm{eV}$. Assuming $T_{i}=T_{e}$, the calculated $f_{G A M}$ is 6.3 to $8.1 \mathrm{kHz}$, slightly lower than the observed mode frequency of $8.5 \mathrm{kHz}$ on the fast probe (see Fig. 2(d)). To test the scaling further, we plot in Fig. 3 the experimentally observed coherent mode frequencies detected at different radii by the fast probe versus the local $T_{e}$ under three different $q(a)$ discharges. In the figure the dashed and dotted line denotes the theoretical prediction of the GAM frequencies at different $T_{e}$ (assuming $T_{i}=T_{e}$ ) for $\gamma=1$ (isothermal) and $\gamma=5 / 3$ (no heat conduction), respectively. Several conclusions can be drawn: (i) In general, the $f_{G A M}$ changes monotonically with the local temperature and resides roughly in the range of the frequency-dispersion predicted by theories. The discrepancy between the theory and experiment seems to be much smaller than those observed in DIII-D [3] and T-10 tokamaks [8]. Moreover, TEXTOR has a circular plasma, and hence, the impact of geometrical factors such as the ellipticity is negligible; (ii) for the three $q(a)$ values investigated, the difference for their influence on the $f_{G A M}$ is almost undistinguishable, as expected from the formula. However, the impact of the safety factor on the level of GAMs does exist, which will be shown in Fig. 7; (iii) in the temperature range of $T_{e} \approx(20-45) \mathrm{eV}$, the variation of $f_{G A M}$ is quite flat, implying that the GAM frequency is common within certain width of the radial region. Whether it is related to the eigenmode characteristics of GAMs $[14,17]$ is still under investigation. 

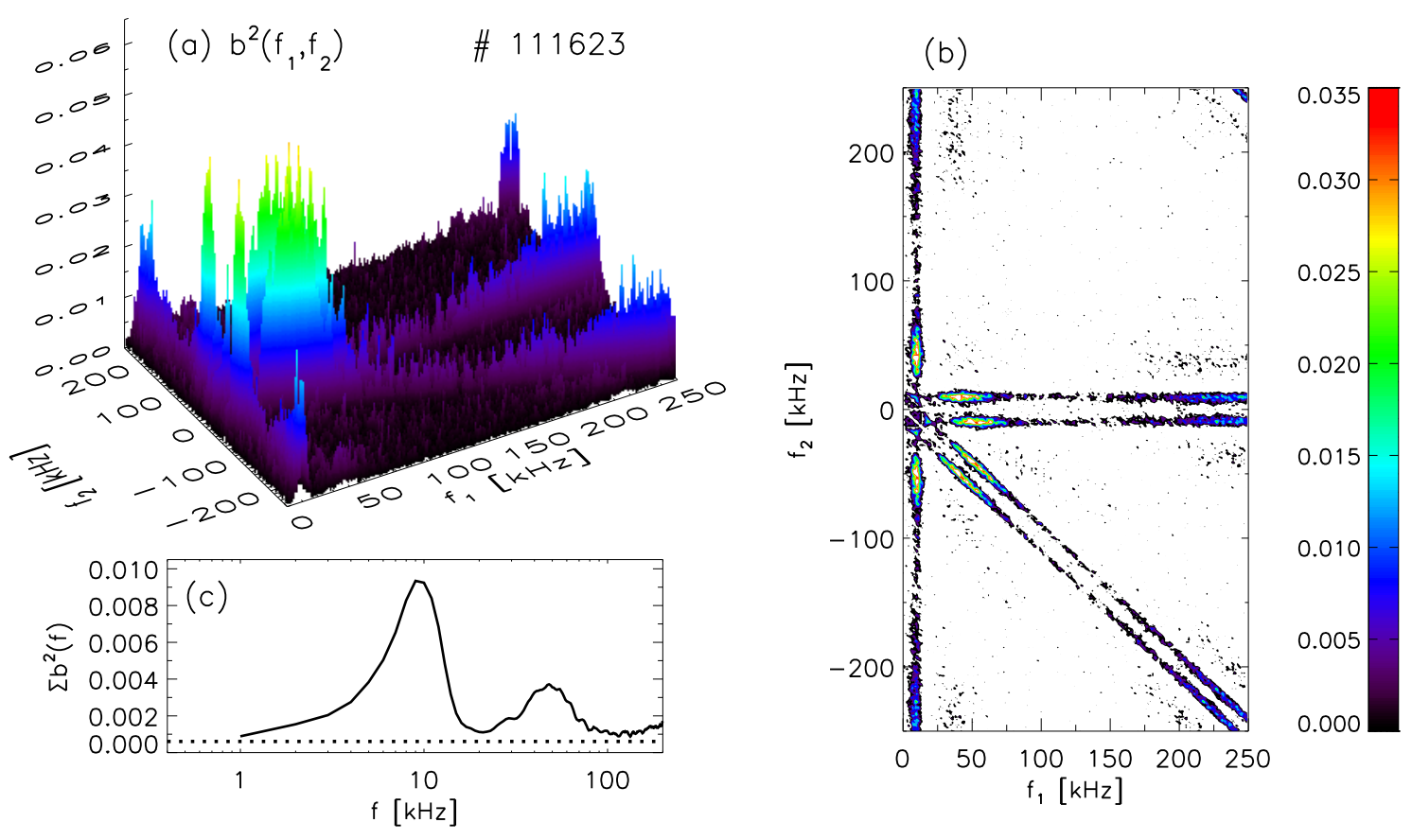

FIG. 4: Results of bispectral analysis. (a) Surface-plot of squared auto-bicoherence in $V_{f}$ fluctuations; (b) contour-plot of squared auto-bicoherence; and (c) summed squared auto-bicoherence as a function of frequency. The dotted line shows the statistical noise level.

According to the theory [1], GAM zonal flows are generated by small-scale fluctuations via nonlinear three-wave interactions under the resonance conditions that $f_{1} \pm f_{2}=f_{3}$ and $\mathbf{k}_{1} \pm \mathbf{k}_{2}=\mathbf{k}_{3}$, where $\left(f_{1}, f_{2}, f_{3}\right)$ and $\left(\mathbf{k}_{1}, \mathbf{k}_{2}, \mathbf{k}_{3}\right)$ are the frequencies and wavenumbers of the three waves. For identifying the three-wave coupling, bispectral analysis technique [22] has been widely applied in many devices $[2,5,9,11]$. The squared bicoherence of fluctuation signals $x(t), y(t)$ and $z(t)$ is defined as $b_{X Y Z}^{2}\left(f_{1}, f_{2}\right)=\left|B_{X Y Z}\left(f_{1}, f_{2}\right)\right|^{2} /\left[\left\langle\left|X\left(f_{1}\right) Y\left(f_{2}\right)\right|^{2}\right\rangle\langle| Z\left(f_{1} \pm\right.\right.$ $\left.\left.f_{2}\right)\left.\right|^{2}\right\rangle$, where $B_{X Y Z}\left(f_{1}, f_{2}\right)=\left\langle X\left(f_{1}\right) Y\left(f_{2}\right) Z^{*}\left(f_{1} \pm f_{2}\right)\right\rangle$ is the bispectrum which measures the degree of phase coherence among modes (* represents a complex conjugate), and $X(f), Y(f)$ and $Z(f)$ are the Fourier transform of $x(t), y(t)$ and $z(t)$, respectively. If $x(t), y(t)$ and $z(t)$ is the same signal it is called auto-bispectrum while for different signals it is called cross-bispectrum. In this study, we have analyzed both the auto-bispectrum in potential fluctuations and the cross-bispectrum between potential and density fluctuations. To optimize the statistics, it is crucial to obtain a large number of realizations. In the present work, we took the fluctuation data from the stationary probe and made ensemble averaging over $\mathrm{M}=1650$ realizations by overlapping $50 \%$ for the neighboring ensembles. In each realiza- 
tion we used 500 data points (=1 ms in length), and consequently, the frequency resolution of the bispectrum is $1 \mathrm{kHz}$. For both auto- and cross-bispectrum, significant bicoherence has been observed between the GAM components and the ambient small-scale turbulence. As an example, in this paper we present the squared auto-bicoherence in $V_{f}$ fluctuations. Figure 4(a) shows the surface-plot of $b^{2}\left(f_{1}, f_{2}\right)$ in the $f_{1}-f_{2}$ plane for the same shot depicted in Fig. 2. The corresponding contour-plot is shown in Fig. 4(b). Because, for the auto-bicoherence $f_{1}$ and $f_{2}$ are interchangeable, the graph is symmetric with respect to the $f_{2}=f_{1}$ line for $f_{2}>0$ and to the $f_{2}=-f_{1}$ line for $f_{2}<0$. Figures $4(\mathrm{a})$ and (b) clearly show that the bicoherence values along the $f_{2}= \pm 9.0 \mathrm{kHz}$ and $f_{2}=-f_{1} \pm 9.0 \mathrm{kHz}$ lines are much larger than those at other frequencies, suggesting a significant level of nonlinear coupling concentrated at these frequencies, i. e., $f_{1}+f_{2}=9.0 \mathrm{kHz}$. In Fig. 4(a), a high level of bicoherence is also shown up around $50 \mathrm{kHz}$, which is usually considered as the quasi-coherent modes in the ambient turbulence. The results imply a strong interaction between these modes and the GAMs as well as other frequency components. To inspect further the bicoherence for all frequencies obeying the selection rule $f=f_{1}+f_{2}$, the summed squared bicoherence $\sum b^{2}(f)=\sum_{f=f_{1}+f_{2}} b^{2}\left(f_{1}, f_{2}\right) / N(f)$ has been computed, where $N(f)$ is the number of Fourier components for each $f$ in the summation. The result is plotted in Fig. 4(c), in which we can see two significant peaks at about $f=9 \mathrm{kHz}$ and $50 \mathrm{kHz}$. The value of $\sum b^{2}(f)$ is much higher than the statistical uncertainty $\approx 1 / M=6.1 \times 10^{-4}$, as indicated by the dotted line. These two peaks are in agreement with those shown in the power spectrum of $V_{f}$ fluctuations (see Fig. 2(d)) and the salient peak at $f \approx 9 \mathrm{kHz}$ is attributed to the GAM zonal flows. Obviously, the highest peak at $f=9 \mathrm{kHz}$ in the $\sum b^{2}(f)$ demonstrates that the GAMs have the strongest nonlinear coupling with broadband ambient turbulence, as expected by the theory [1]. Meanwhile it is noted that the quasi-coherent modes at $f \approx 50 \mathrm{kHz}$ also involve strong nonlinear interactions with background turbulence.

From all above facts, i. e, the $m=n=0$ mode structure in $\tilde{V}_{f}$, the frequency dependence on the local temperature and the nonlinear interactions with broadband turbulence, we conclude that the observed long-distance correlations in $\tilde{V}_{f}$ are essentially attributed to GAM zonal flows.

In our experiments, the fast probe changes its radial position $\left(r_{2}\right)$ relative to the stationary probe $\left(r_{1}\right)$ in every shot. Such a setting is, in principle, capable of inferring the spatial correlation structure (toroidal + radial) of GAMs through their cross-correlation function at 

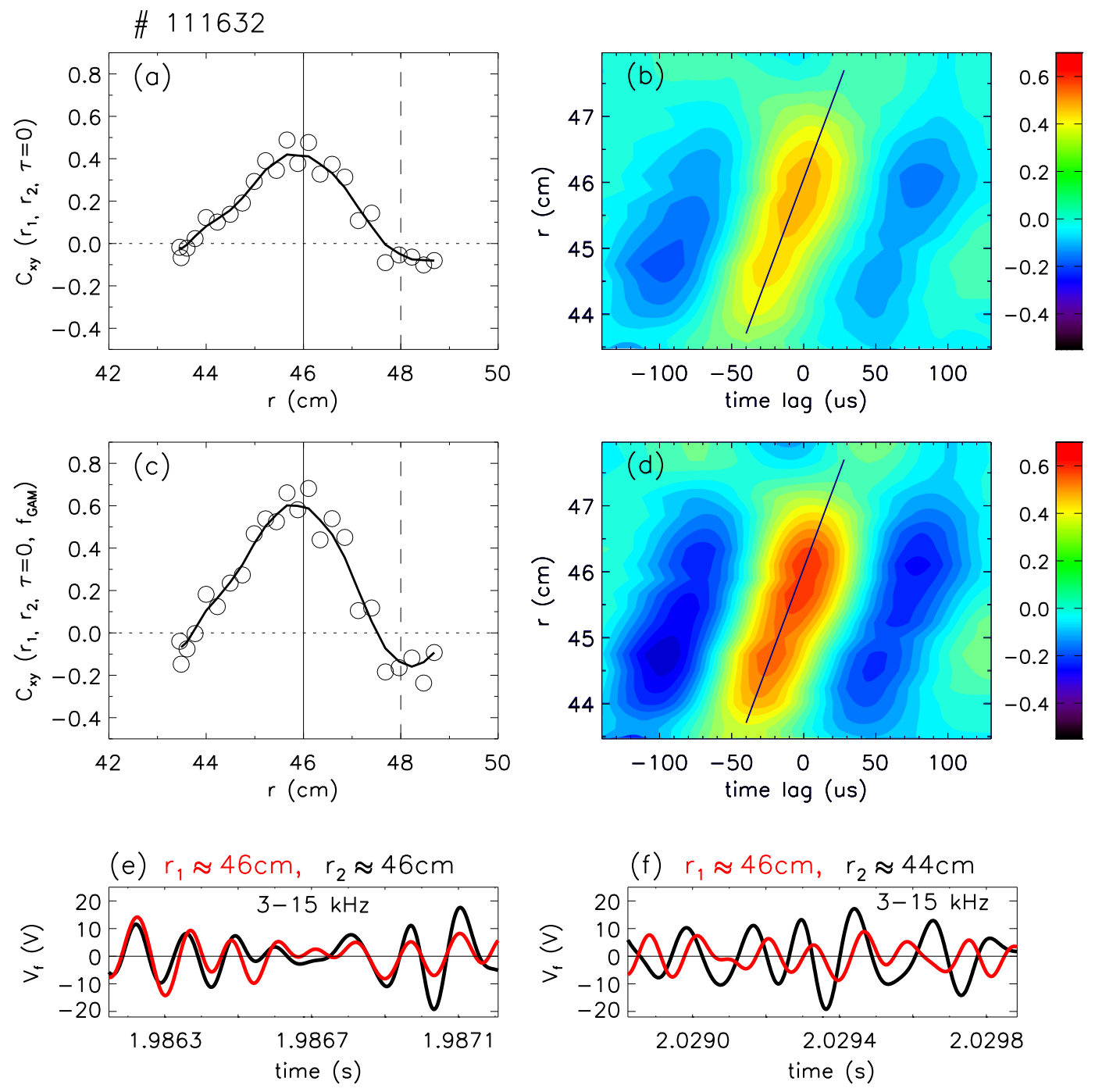

FIG. 5: (a) The radial structure of GAM correlations, estimated from the cross-correlation of $\tilde{V}_{f}$ signals at a zero-delay time $\left[C_{x y}\left(r_{1}, r_{2}, \tau=0\right)\right]$ measured by two toroidally distant probes. The vertical dashed line denotes the limiter position; (b) contour-plot of cross-correlation $\left(C_{x y}\right.$ on $\tilde{V}_{f}$ ) showing the radially outward propagation of GAMs at a speed of $V_{r} \approx 0.6 \mathrm{~km} / \mathrm{s}$; (c) the radial structure of GAM correlation, estimated from the cross-correlation of $\tilde{V}_{f}$ signals in the GAM frequency range of $3-15 \mathrm{kHz}$ at a zero-delay time $\left[C_{x y}\left(r_{1}, r_{2}, \tau=0, f_{G A M}\right)\right]$ measured by two toroidally distant probes; (d) contour-plot of cross-correlation $C_{x y}$ on $\tilde{V}_{f}$ in the GAM frequency range of 3-15 kHz; (e) waveforms of $\tilde{V}_{f}$ on two toroidally distant probes (the black is for the fast probe and the red is for the stationary probe) around the same radial position; (f) waveforms of $\tilde{V}_{f}$ on two toroidally distant probes at two different radial positions. The signals in (e) and (f) are filtered in the frequency range of $3-15 \mathrm{kHz}$. 
a zero delay time, $C_{x y}\left(r_{1}, r_{2}, \tau=0\right)$. Since GAMs have a uniform toroidal structure $\left(k_{\phi} \rightarrow 0\right)$, the cross-correlation coefficient measured by the two toroidally separated probes actually reflects the radial structure of the GAM correlations. Note that for a simple harmonic wave of $A \sin (k r-\omega t), C_{x y}\left(r_{1}, r_{2}, \tau=0\right) \equiv \cos \left[k\left(r_{1}-r_{2}\right)\right]$. As seen in Fig. 2, the GAM zonal flow has relatively narrow frequency and wavelength bands. Therefore, the spatial variation of $C_{x y}\left(r_{1}, r_{2}, \tau=0\right)$ versus the radial position of the moving probe $\left(r_{2}\right)$ is an indication of the spatial phase structure of the GAMs. Figure 5(a) displays the correlation coefficient as a function of radial locations of the fast probe while the stationary one is fixed at $r_{1} \approx 46 \mathrm{~cm}$ (indicated by the vertical solid line). As we expected, the maximum $C_{x y}\left(r_{1}, r_{2}, \tau=0\right)$ is seen when two probes are approximately at the same radial locus. The value of the coefficient drops when $r_{2}$ is getting away from $r_{1}$. Because the deepest position of the fast probe is limited to avoid overheating on probe pins, the radial profile of $C_{x y}\left(r_{1}, r_{2}, \tau=0\right)$ is bounded at $r \approx 43.5 \mathrm{~cm}$. Nevertheless, the profile shows a sinusoidally changing structure with $\lambda_{r} \simeq 9 \mathrm{~cm}$ ( or $k_{r} \simeq 0.7 \mathrm{~cm}^{-1}$ ). A similar sinusoidal structure of low-frequency zonal flows has been observed in CHS stellarator [23]. As we depicted already in Fig. 2(c), the GAM zonal flow displays a propagation in the radial outward direction. In order to estimate the propagating velocity, we convert the time trace of the fast probe to radial positions. Figure 5(b) plots the contour of the cross-correlation as a function of radius and time lag. On can see that the GAM structure is moving radially outward $(\delta r)$ along the black line with increasing time lag $(\delta \tau)$. The propagation phase speed is thus deduced as $V_{r}=\delta r / \delta \tau \approx 0.6 \mathrm{~km} / \mathrm{s}$. In this discharge, the local GAM frequency at $\mathrm{r}=46 \mathrm{~cm}$ is $f_{G A M} \approx 5.8 \mathrm{kHz}$. If one takes $\lambda_{r} \simeq 9 \mathrm{~cm}$ at that location, the local phase velocity of the GAM is $V_{p h, r}=f_{G A M} \times \lambda_{r}=0.52 \mathrm{~km} / \mathrm{s}$, close to the $V_{r}$ derived from the contour-plot.

In Fig. 2(e) we showed that the inclusion of ambient turbulence undermines the magnitude of GAM correlations. To make a more clear picture for the GAM propagation, we have also calculated the cross-correlation using the fluctuation data of GAMs only (band-passed in $3-15 \mathrm{kHz})$. The results of $C_{x y}\left(r_{1}, r_{2}, \tau=0, f_{G A M}\right)$ and the contour-plot of $C_{x y}\left(\tau, f_{G A M}\right)$ on $\tilde{V}_{f}$ are shown in Figs. 5(c) and (d), respectively. Compared to Figs. 5(a) and (b), the $C_{x y}$ values in Figs. 5(c) and (d) become much larger using the filtered data. However, both the radial correlation length and the phase speed of the GAMs are nearly the same in both cases. For a better understanding on the spatial variation of the GAM correlation structure displayed in Fig. 5(a, c), we inspect the temporary behavior and phase relation of the GAM 
signals (band-passed in $3-15 \mathrm{kHz}$ ) detected by the two probes at different radii. In Fig. 5(e) we see synchronized oscillations of the zonal potentials when the two probes are around the same flux surface. When the fast probe moves to $r_{2}=44 \mathrm{~cm}$ while the reference one stays at $r_{1}=46 \mathrm{~cm}$, Fig. 5(f) clearly shows a phase shift between the two signals. The waveform on the fast probe leads about $\pi / 2$ phase to that on the stationary probe, implying a quarter wavelength $\left(\lambda_{r} / 4\right)$ phase shift in space, which is in good agreement with that measured in Fig. 5(a) or (c). These results indicate that the radial variation of the GAM correlations is mostly caused due to the propagation of the GAM structure.

It should be noted that, up to now, the radial propagation properties of the GAMs have not yet been described explicitly by theories. In many literatures [1, 12, 13, 24], the GAMs are simply described as standing waves with a frequency-dispersion relation

$f_{G A M} \simeq C_{s} \sqrt{2+1 / q^{2}} / 2 \pi R$ without information of wavenumbers. In recent work, the radial propagation of GAMs and GAM eigenmodes have been proposed by several authors [14-18]. However, no general rules are provided for determining the direction and velocity of the GAM propagation. Experimental reports on this aspect are also rare. In the HL-2A tokamak [11], the statistical analysis shows a single positive peak at GAM frequencies in the radial wavenumber spectrum $S\left(k_{r}\right)$, suggesting an outward radial propagation of the GAMs. Meanwhile they observed almost equal group and phase velocities of $\sim 0.25 \mathrm{~km} / \mathrm{s}$ with a nearly linear dispersion relation $\bar{k}_{r}(f)$. The GAM propagating velocities observed in the HL-2A are a bit lower than ours. Another result has been reported from the JFT-2M tokamak [7], in which both plus and minus phase delay $\left(\propto k_{r}\right)$ of GAMs were seen in different radial locations, suggesting a non-uniform propagation of GAMs in both outward and inward directions. In the TJ-II stellarator, a sign of radially propagating zonal flows (below $20 \mathrm{kHz}$ ) has also been mentioned [19]. In addition to above results, our observations provide direct and explicit evidence on the GAM propagation, which could be one of important characteristics of the GAM zonal flows.

\subsection{Influence of local plasma parameters on the radial propagation of GAMs}

In order to investigate further the possible impact of local plasma parameters on the radial structure and propagation of GAMs, we made a parametric study in TEXTOR ohmic discharges by stepwise increasing the plasma density approaching to the density-limit un- 


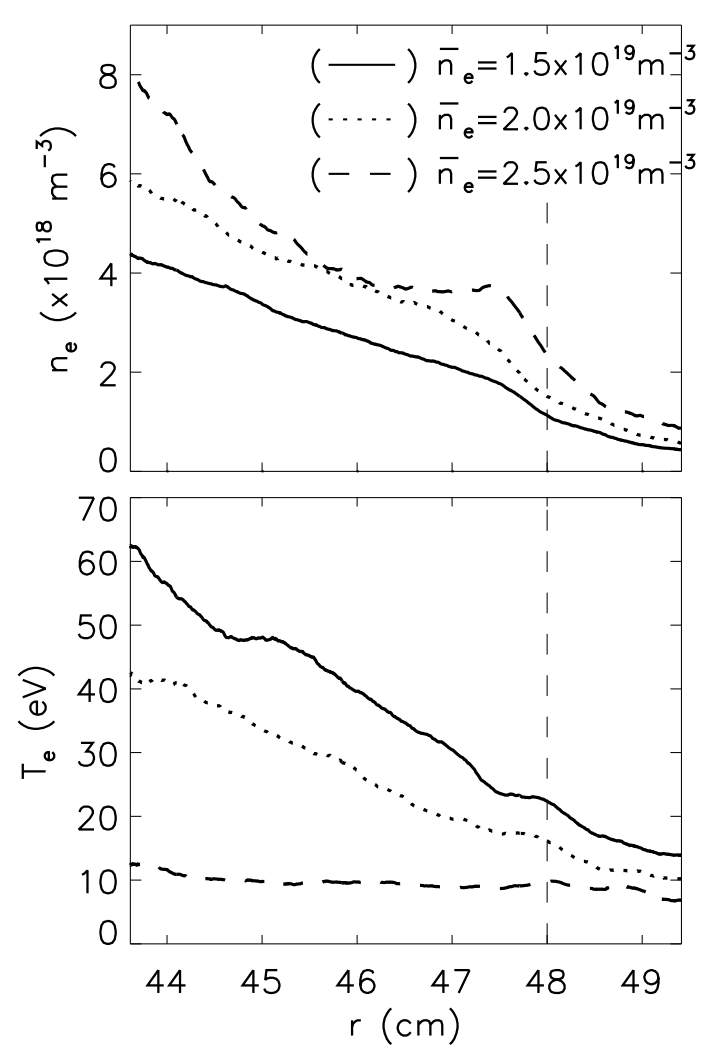

FIG. 6: Radial profiles of edge density (top) and electron temperature (bottom) in different $\bar{n}_{e}$ discharges in the density scan experiment at TEXTOR. The vertical dashed line denotes the limiter position.

der two different edge safety factors $(q(a)=5.9$ and 5.0). For $q(a)=5.9$, we scanned the density in a range of $\bar{n}_{e}=(1.5-3.0) \times 10^{19} \mathrm{~m}^{-3}$ while at the last density the plasmas disrupted. Therefore, we obtained the available edge $n_{e}$ and $T_{e}$ profiles for $\bar{n}_{e}=1.5,2.0$ and $2.5 \times 10^{19} \mathrm{~m}^{-3}$ discharges, as plotted in Fig. 6. The figure clearly shows that with increasing $\bar{n}_{e}$ the edge density gradually increases whereas the edge $T_{e}$ strongly decreases because of cooling effects. The corresponding radial structures of GAM correlations are plotted in Fig. 7. Here and hereafter, the GAM correlations are all computed using band-passed $\tilde{V}_{f}$ data $(3-20 \mathrm{kHz})$ for getting clear GAM pictures. In low density cases $\left(\bar{n}_{e} \leq 2.0 \times 10^{19} \mathrm{~m}^{-3}\right)$, the $C_{x y}\left(r_{1}, r_{2}, \tau=0, f_{G A M}\right)$ profile varies little although the local $n_{e}$ and $T_{e}$ in the plasma edge both change remarkably (see Fig. 6). However, at higher densities when the $\bar{n}_{e}$ increases from 2.0 to $2.5 \times 10^{19} \mathrm{~m}^{-3}$, the magnitude of GAM correlations largely decrease. In a similar way, we made a density scan for $q(a)=5.0$ and finally we got available edge profiles for $\bar{n}_{e}=2.5$ and $3.0 \times 10^{19} \mathrm{~m}^{-3}$ discharges. The radial dependences of the GAM correlations are 
also shown in Fig. 7, where we can see a large reduction in the magnitude of GAM correlations as $\bar{n}_{e}$ increases from 2.5 to $3.0 \times 10^{19} \mathrm{~m}^{-3}$. Comparing the profiles of two different $q(a)$ at the same $\bar{n}_{e}=2.5 \times 10^{19} \mathrm{~m}^{-3}$ (see yellow and green curves), we can see that the magnitude of the GAM correlations is much lower in the higher $q(a)$, suggesting an influence of the safety factor on the level of GAMs. In figure 7 the radial profiles of the GAM correlation are not symmetric about the reference probe at $r \approx 46 \mathrm{~cm}$. This could be caused by the slight change of GAM frequencies at various radial locations on both sides of the reference probe. In addition, the strong interaction between GAMs and ambient turbulence modulates the GAM amplitudes individually at different radial locations, which may also cause deformation of the symmetry. Nevertheless, the radial correlations reflect the spatial structure of the GAMs, as illustrated in Fig. 5. From Fig. 7 we see that the parametric impact on the GAM wavelength $\left(\lambda_{r}\right)$ appears quite small. The $\lambda_{r}$ only decreases slightly in higher $\bar{n}_{e}$ and $q(a)$ discharges. By extrapolating the radial profiles toward the core region, we can get $\lambda_{r} / 2$ values changed roughly from 4.5 to $6.5 \mathrm{~cm}$, i. e., $\lambda_{r} \approx(9-13) \mathrm{cm}$ and $k_{r} \approx(0.5-0.7) \mathrm{cm}^{-1}$, in those discharges. Recently, a similar correlation structure of GAMs with about the same $4 \mathrm{~cm}$ wide peak distribution at the inner edge region of TEXTOR has been observed by the beam-emission-spectroscopy [25].

Then, we surveyed the impact of $\bar{n}_{e}$ and $q(a)$ on the radial propagation of GAMs. It has been found that the $q(a)$ affects very little on the radial propagation of GAMs, whereas the plasma density brings strong effects on the GAM propagating speed. Figure 8 plots the contour-plot of the GAM propagation, $C_{x y}\left(\tau, r, f_{G A M}\right)$, in various $\bar{n}_{e}$ discharges $(5.0 \leq$ $q(a) \leq 5.9)$. One can clearly see that with increasing $\bar{n}_{e}$ from 1.5 to $3.0 \times 10^{19} \mathrm{~m}^{-3}$, the radial propagating velocity, $V_{r}$, is gradually reduced from 2.0 to $0.6 \mathrm{~km} / \mathrm{s}$, as indicated in the figure caption.

To gain more insight into the damping effects of enhanced $\bar{n}_{e}$ on the $V_{r}$, we compared the $V_{r}$ values deduced from the "global" contour-plot with the local GAM phase velocity, $f_{G A M} \times \lambda_{r}$, measured around $r \approx 46 \mathrm{~cm}$. The results are listed in table I. Given in the first row are the $V_{r}$ values estimated in Fig. 8. The second and third rows record the local $n_{e}$ and $T_{e}$ values measured at $r \approx 46 \mathrm{~cm}$ by the fast probe array in various $\bar{n}_{e}$ discharges (see Fig. 6). The corresponding GAM frequencies at that location are listed in the fourth row, and the fifth are the $\lambda_{r}$ values estimated from the $C_{x y}\left(r_{1}, r_{2}, \tau=0, f_{G A M}\right)$ profiles drawn in Fig. 7. The local GAM phase velocities, $f_{G A M} \times \lambda_{r}$, are listed in the bottom tables. The 


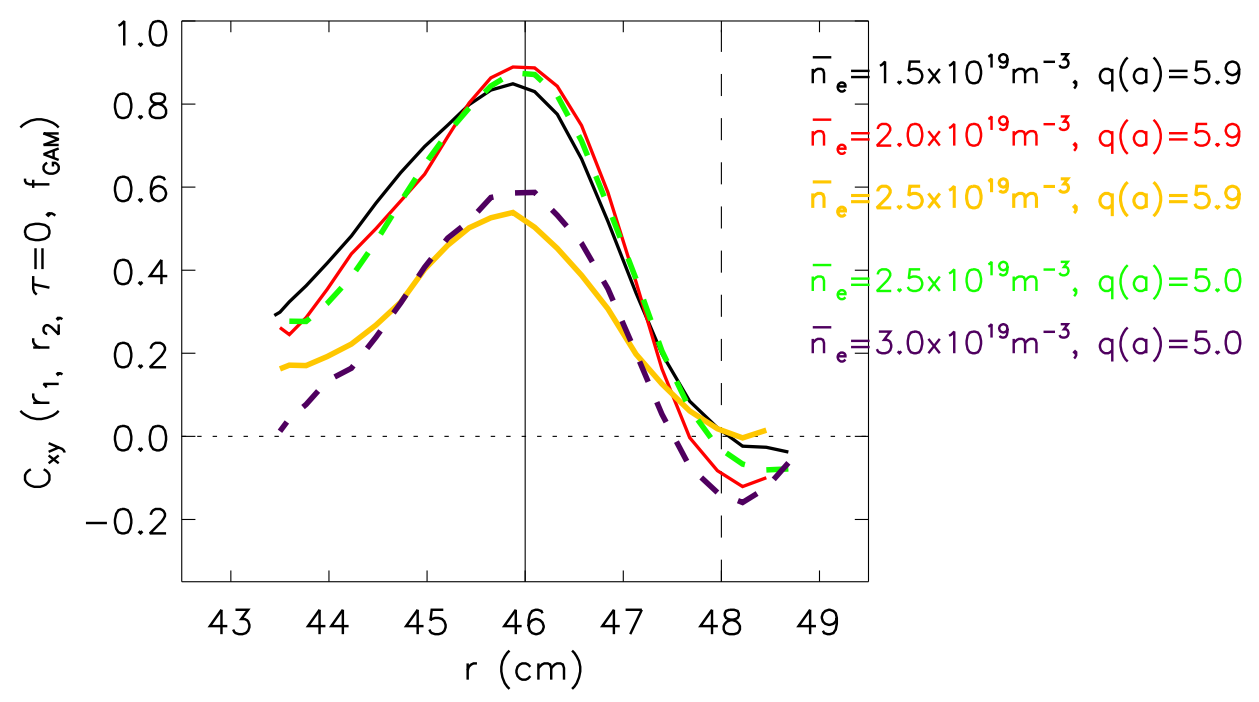

FIG. 7: Radial structures of GAM correlations, estimated from the cross-correlation of $\tilde{V}_{f}$ signals in the GAM frequency range of $3-20 \mathrm{kHz}$ at a zero-delay time, $C_{x y}\left(r_{1}, r_{2}, \tau=0, f_{G A M}\right)$, in different $\bar{n}_{e}$ discharges under two safety factors $[q(a)=5.0$ and 5.9]. The vertical dashed line denotes limiter position.

local phase speeds are slightly lower than the global $V_{r}$, most likely due to the error between local and global estimations, e. g., the GAM propagates radially but varies in frequency and amplitude at different loci. But those two values are quite close, verifying that the observed GAM propagation is dominated by the GAM phase velocity. In the table, we can see that with increasing $\bar{n}_{e}$ the local plasma temperature is reduced, resulting in a decrease in the GAM frequency. As a consequence, the radial propagating speed of GAMs is moderated. The results also testify the theoretical prediction of the GAM frequency dependence on the local temperature.

The mechanisms governing the radial propagation of GAMs remain unclear. In the above density-limit discharges, the increase of plasma density induces an increase in edge $n_{e}$ and a decrease in edge $T_{e}$, and thus an increase of the collisionality, which may exhaust the GAM wave energy and moderate its group velocity. The magnitude of the group velocity and its quantitative relation with the phase velocity are not clear yet in the present studies. The propagation of GAMs may also be related to the turbulence spreading along the radial direction [26]. Assuming that the turbulence spreading is a general phenomenon, the GAM zonal flows may also spread out radially. To unravel these underlying physics, input from 
(a) $\bar{n}_{e}=1.5 \times 10^{19} \mathrm{~m}^{-3}$

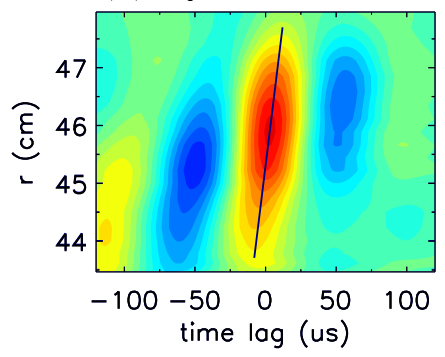

(b) $\overline{\mathrm{n}}_{\mathrm{e}}=2.0 \times 10^{19} \mathrm{~m}^{-3}$

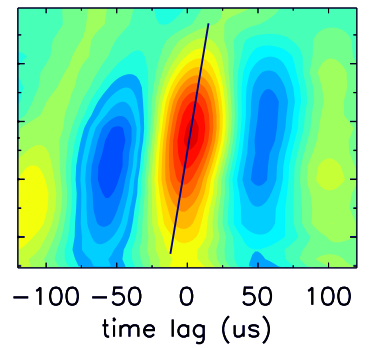

(c) $\bar{n}_{e}=2.5 \times 10^{19} \mathrm{~m}^{-3}$

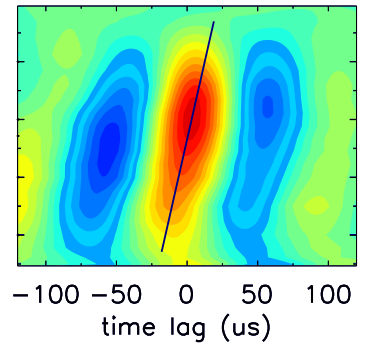

(d) $\bar{n}_{e}=3.0 \times 10^{19} \mathrm{~m}^{-3}$

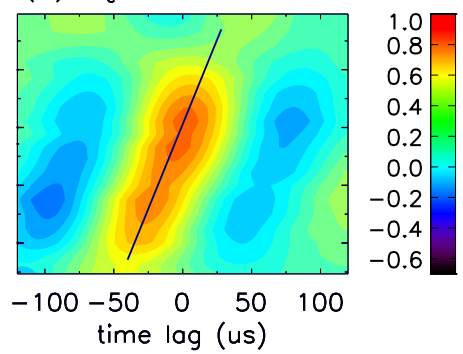

FIG. 8: Contour-plot of the radial propagation of GAMs in various $\bar{n}_{e}$ discharges for a safety factor range of $5.0 \leq q(a) \leq 5.9$. The radially moving speed of the GAMs is approximately (a) $V_{r} \approx 2.0 \mathrm{~km} / \mathrm{s} ;$ (b) $1.5 \mathrm{~km} / \mathrm{s} ;$ (c) $1.1 \mathrm{~km} / \mathrm{s}$ and (d) $0.6 \mathrm{~km} / \mathrm{s}$.

TABLE I: Comparison between experimentally observed radially propagating speed $\left(V_{r}\right)$ of GAMs in Fig. 8 with the local GAM phase velocity, $f_{G A M} \times \lambda_{r}$, estimated by locally detected plasma parameters around $r \approx 46 \mathrm{~cm}$.

\begin{tabular}{|c|c|c|c|c|c|}
\hline $\begin{array}{c}\text { Observed radial } \\
\text { propagation of GAMs }\end{array}$ & $\begin{array}{c}\mathbf{V}_{\mathbf{r}} \\
(\mathrm{km} / \mathrm{s})\end{array}$ & 2.0 & 1.5 & 1.1 & 0.6 \\
\hline \multirow{4}{*}{$\begin{array}{l}\text { Local plasma } \\
\text { parameters } \\
\text { at } r \approx 46 \mathrm{~cm}\end{array}$} & $\begin{array}{c}\mathbf{n}_{\mathbf{e}} \\
\left(10^{18} m^{-3}\right)\end{array}$ & 2.5 & 3.9 & 4.1 & \begin{tabular}{|l|l}
4.3 \\
\end{tabular} \\
\hline & $\begin{array}{r}\mathbf{T}_{\mathbf{e}} \\
(\mathrm{eV})\end{array}$ & 42 & 29 & 25 & 9.8 \\
\hline & $\begin{array}{r}f_{\mathrm{GAM}} \\
(\mathrm{kHz}) \\
\end{array}$ & 10.0 & 8.9 & 8.5 & 5.8 \\
\hline & $\begin{array}{c}\lambda_{\mathbf{r}} \\
(\mathrm{cm})\end{array}$ & 13 & 11.5 & 10 & 9 \\
\hline $\begin{array}{l}\text { Phase velocity } \\
\text { at } r \approx 46 \mathrm{~cm}\end{array}$ & $\begin{array}{c}f_{\text {GAM }} \times \lambda_{\mathbf{r}} \\
(\mathrm{km} / \mathrm{s})\end{array}$ & 1.3 & 1.0 & 0.85 & 0.52 \\
\hline
\end{tabular}

theories and simulations would be very interesting.

\section{CONCLUSION}

In conclusion, we reported the experimental observation of GAM zonal flows at the edge of TEXTOR toakamk using two toroidally separated Langmuir probe arrays. The GAMs are observed in potential fluctuations with symmetric toroidal and poloidal structures and a radial extent of $k_{r} \simeq(0.5-0.7) \mathrm{cm}^{-1}$. The GAM frequency, $f_{G A M}$, shows a dependence on the local temperature, consistent with theoretical predictions. Bispectral analysis indicates strong nonlinear interactions between the GAMs and ambient turbulence. Moreover, 
the GAM packet exhibits explicitly a radially outward propagation. The radial correlation structure of GAMs and their radial propagation have been investigated in various plasma density and safety factor discharges. The magnitude of the GAM correlations reduces remarkably with the increase of the plasma density as approaching to the density-limit, while the radial wavelength of GAMs only decreases slightly in higher density and larger $q(a)$ discharges. With increasing plasma density, the radial propagating phase speed of GAMs is strongly reduced along with the drop of the local temperature. The results therefore provide new evidence on the propagation properties of GAM zonal flows.

\section{ACKNOWLEDGEMENTS}

The authors thank Mr. S. Kraus and Mr. M. Berte for maintenance of the probe measurements at TEXTOR.

[1] P. H. Diamond et al., Plasma Phys. Controlled Fusion 47, R35 (2005).

[2] M. Shats and W. Solomon, New J. Phys. 4, 30 (2002).

[3] G. R. Mckee et al., Phys. Plasmas 10, 1712 (2003).

[4] Y. Hamada et al., Nucl. Fusion 45, 81 (2005).

[5] Y. Nagashima et al., Phys. Rev. Lett. 95, 095002 (2005).

[6] G. D. Conway et al., Plasma Phys. Controlled Fusion 47, 1165 (2005).

[7] T. Ido et al., Plasma Phys. Controlled Fusion 48, S41 (2006).

[8] A. V. Melnikov et al., Plasma Phys. Controlled Fusion 48, S87 (2006).

[9] K. J. Zhao et al., Phys. Rev. Lett. 96, 255004 (2006).

[10] A. Krämer-Flecken et al., Phys. Rev. Lett. 97, 045006 (2006).

[11] T. Lan et al., Plasma Phys. Controlled Fusion 50, 045002 (2008).

[12] N. Winsor et al., Phys. Fluids 11, 2448 (1968).

[13] A. B. Hassam and J. F. Drake, Phys. Fluids B 5, 4022 (1993).

[14] K. Itoh et al., Plasma Fusion Res. 1, 037 (2006).

[15] F. Zonca and L. Chen, Europhys. Lett. 83, 35001 (2008).

[16] X. Q. Xu et al., Phys. Rev. Lett. 100, 215001 (2008). 
[17] Z. Gao et al., Phys. Plasmas 15, 072511 (2008).

[18] R. Hager and K. Hallstschek, Phys. Plasmas 16, 072503 (2009).

[19] C. Hidalgo et al., Europhys. Lett. 87, 55002 (2009).

[20] K. Hallatschek, Plasma Phys. Controlled Fusion 49, B137 (2007).

[21] Y. Xu et al., Nucl. Fusion 47, 1696 (2007).

[22] Y. Kim and E. Powers, IEEE Trans. Plasma Sci. 7, 120 (1979).

[23] A. Fujisawa et al., Phys. Rev. Lett. 93, 165002 (2004).

[24] K. Itoh et al., Plasma Phys. Controlled Fusion 47, 451 (2005).

[25] S. Zoletnik, private communication (2011).

[26] T. Estrada et al., Nucl. Fusion 51, 032001 (2011). 